\title{
The role of echocardiography and doppler ultrasound in evaluation of prosthetic mitral valves
}

\section{(DDiana Rudan* (DIvana Jurin}

University Hospital Dubrava, Zagreb, Croatia
KEYWORDS: mitral regurgitation, mitral valve repair, mitral valve replacement, echocardiography. CITATION: Cardiol Croat. 2018;13(5-6):182. | https://doi.org/10.15836/ccar2018.182

*ADDRESS FOR CORRESPONDENCE: Ivana Jurin, Klinička bolnica Dubrava, Av. Gojka Šuška 6, HR-10000 Zagreb, Croatia. / E-mail: drudan3@yahoo.com

ORCID: Diana Rudan, https://orcid.org/0000-0001-9473-2517 • Ivana Jurin, https://orcid.org/0000-0002-2637-9691

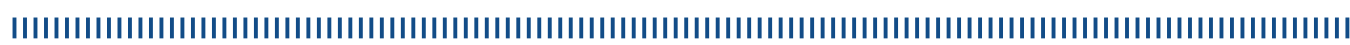

Mitral regurgitation (MR) is characterized by abnormal backflow of blood through the mitral valve during the systolic phase of cardiac cycle. Usually, it is the main indication for mitral valve repair or mitral valve replacement at the onset of symptoms of congestive heart failure. Although mitral valve repair is now frequently performed, especially for mitral regurgitation, valve replacement remains common ${ }^{1}$.

Echocardiography with Doppler is the method of choice for the non-invasive evaluation of prosthetic valve function and can provide a valuable information about functioning of operating valve and about medical management and considerations for reoperation on valvular complications ${ }^{2}$.

Because the assessment of prosthetic valve is more demanding, both to perform and to interpret, compared with native valves, transesophageal echocardiography (TEE) is preferable method for the evaluation of prosthetic valvular structure and associated complications ${ }^{3,4}$.
RECEIVED:

May 8, 2018

ACCEPTED:

May 10, 2018
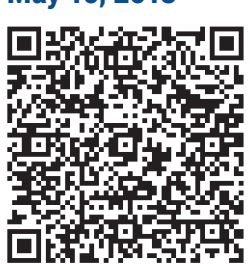

$\square$ Cardiologia Croatica 2018;13(5-6):182. 